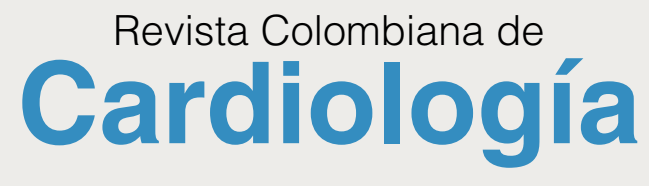

www.elsevier.es/revcolcar

ARTÍCULO ESPECIAL

\title{
Anticoagulantes orales directos para el tratamiento de los pacientes con fibrilación auricular no valvular
} CrossMark

\section{Direct oral anticoagulants for the treatment of patients with non-valvular atrial fibrillation}

\author{
Mauricio Duque-Ramírez ${ }^{a, b, c, *}$, Juan Carlos Díaz-Martínez ${ }^{a, b, c}$, \\ Julián Miguel Aristizábal-Aristizábal ${ }^{a, b, c, d}$, Jorge Enrique Velásquez-Vélez ${ }^{a, b, c, e, f}$, \\ Jorge Eduardo Marín-Velásquez ${ }^{a, b, c}$ y William Uribe-Arango ${ }^{a, b, f}$
}

\author{
a Universidad CES, Medellín, Colombia \\ b CES Cardiología, Clínica CES, Medellín, Colombia \\ c Servicio de Electrofisiología, Clínica Las Américas, Medellín, Colombia \\ ' Clínica del Rosario, Medellín, Colombia \\ e Clínica Somer, Rionegro, Colombia \\ ${ }^{f}$ Centros Especializados de San Vicente Fundación, Rionegro, Colombia
}

Recibido el 3 de octubre de 2016; aceptado el 6 de octubre de 2016

Disponible en Internet el 8 de noviembre de 2016

\section{Introducción}

La fibrilación auricular es la arritmia prevalente más común en el mundo y en vista de que afecta principalmente a personas de edad avanzada se espera que su prevalencia aumente a medida que la población envejece ${ }^{1}$. Adicionalmente, es una causa importante de morbilidad y mortalidad, consultas a urgencias y aumento del uso de los sistemas de salud y buena parte de estos costos se asocian con el incremento en el riesgo de ataques cerebro-vasculares. Desde la década de 1970, por medio de la cohorte de Framingham, se logró demostrar que la presencia de fibrilación auricular aumenta cinco veces el riesgo de sufrir un ataque cerebro-vascular ${ }^{1,2}$, y que este fenómeno está estrechamente relacionado con diversos factores, entre ellos la edad

\footnotetext{
* Autor para correspondencia.

Correo electrónico: mauricioduque@une.net.co (M. Duque-Ramírez).
}

y patologías que son más comunes en la población que envejece (hipertensión arterial, diabetes mellitus, falla cardíaca, enfermedad vascular $)^{3,4}$. Estudios realizados a finales de la década de los 80 y principios de los 90, lograron demostrar el beneficio de la warfarina en el manejo de pacientes con fibrilación auricular para disminuir de manera significativa el riesgo de embolia, llegando a convertirse en el estándar de manejo ${ }^{5-9}$. Pese a las dificultades para mantener el INR en un rango estrecho (entre 2 y 3 ) debido a sus múltiples interacciones medicamentosas y alimenticias, aún en grupos especializados el tiempo en rango terapéutico (TRT) es apenas del $60 \%{ }^{10}$. La aparición de nuevos anticoagulantes orales directos (NACO) basados en la inhibición de la trombina (dabigatrán) o el factor Xa (rivaroxabán, apixabán, edoxabán, betrixabán) amplía las opciones terapéuticas para millones de pacientes con fibrilación auricular no valvular.

En esta sección se revisan las propiedades farmacológicas y farmacodinámicas de los NACO disponibles en el mercado mundial en la actualidad (dabigatrán, rivaroxabán, apixabán 
y edoxabán) y se brindan recomendaciones respecto a su uso apropiado seguidas por niveles de evidencia. Los fármacos se presentan en el orden en el cual fueron publicados los estudios que avalan su uso en fibrilación auricular y de ninguna manera el orden de presentación denota una preferencia por alguno de los medicamentos. La correcta selección del fármaco es una decisión que atañe al médico tratante basado en la mejor evidencia disponible y la situación clínica de cada paciente.

\section{Dabigatrán etexilato}

El dabigatrán etexilato y sus glucurónidos acílicos son inhibidores competitivos directos y altamente selectivos de la trombina, la cual desempeña un papel mayor en la cascada de la coagulación y en el desarrollo de trombos, sin efecto sobre otros agentes estimulantes de plaquetas como el ácido araquidónico, el colágeno o el difosfato de adenosina $^{11}$. No obstante, dado su efecto sobre la trombina el dabigatrán se asocia con un efecto inhibitorio más potente en la agregación plaquetaria que los inhibidores del factor Xa (rivaroxabán y apixabán). La forma oral (dabigatrán etexilato) es por sí misma fisiológicamente inactiva, siendo una prodroga del dabigatrán, la porción activa en el plasma ${ }^{11}$. Después de una dosis oral, el dabigatrán etexilato se metaboliza en forma rápida y completa (catalizado por carboxilesterasas microsomales) a través de dos intermediarios (BIRB 951 y BIRB 1087) a dabigatrán, con una biodisponibilidad absoluta de aproximadamente $6,5 \%$; la coadministración de dabigatrán etexilato con las comidas retrasa, pero no tiene ningún efecto en la absorción final del medicamento. El máximo efecto anticoagulante ocurre 90 a 120 minutos después de la administración del dabigatrán etexilato y los efectos son aún discernibles al final de un periodo entre 8 y 17 horas de la prescripción ${ }^{12}$. El dabigatrán se distribuye moderadamente a nivel tisular, con un volumen de 60-70 L. Aproximadamente el 35\% del dabigatrán se encuentra ligado a proteínas plasmáticas. Es eliminado primariamente por vía renal $(80 \%)$ y vía heces $(5 \%)$. La concentración de dabigatrán es elevada en ancianos en comparación con jóvenes, incremento que está correlacionado con la reducción en la depuración de creatinina asociada con la edad. El daño hepático moderado no parece tener impacto clínico significativo en los parámetros farmacocinéticos de dabigatrán. El perfil farmacocinético del dabigatrán etexilato ha sido estudiado en voluntarios sanos, pacientes con fibrilación auricular, y/o con daño hepático y/o renal ${ }^{13}$. Los pacientes con fibrilación auricular demuestran un perfil farmacocinético similar a aquel observado en voluntarios sanos, con un estado estable del medicamento obtenido a los cuatro días de iniciada la administración del mismo. Fue el primer DOAC aprobado en los Estados Unidos, Europa y Colombia para uso en fibrilación auricular.

No es un inhibidor o inductor del citocromo P 450, pero sí es sustrato para el transportador de glicoproteína $P$, de tal manera que medicamentos como ketoconazol, verapamilo, amiodarona, quinidina y claritromicina pueden alterar su farmacocinética y aumentar la concentración del fármaco. El incremento en la concentración de dabigatrán inducido por amiodarona es mitigado por el aumento en el aclaramiento renal ${ }^{14}$.

\section{Eficacia terapéutica para la prevención de ataque cerebro-vascular y embolia sistémica en fibrilación auricular}

$\mathrm{Ha}$ sido investigada en distintos estudios multicéntricos, aleatorizados, parcialmente ciegos, controlados contra warfarina: PETRO (fase ॥) y RE-LY (fase III).

\section{Estudio PETRO ${ }^{15}$}

Los participantes recibieron warfarina (INR 2 - 3) sola o dabigatrán etexilato $50,150,300 \mathrm{mg}$ dos veces al día (solo) o con ácido acetil salicílico (ASA) 81 - $325 \mathrm{mg}$ una vez al día por 12 semanas $(n=502)$. Aquellos que recibieron la dosis de $300 \mathrm{mg}$ dos veces al día tuvieron mayor sangrado mientras que los que recibieron la dosis de $150 \mathrm{mg}$ una vez al día tuvieron mayor tasa de eventos embólicos. Fueron cambiados a $300 \mathrm{mg}$ una vez al día y $150 \mathrm{mg}$ dos veces al día, disminuyéndose así la tasa de eventos adversos.

\section{Estudio RE-LY ${ }^{16}$}

Un total de 18.113 pacientes con fibrilación auricular y alto riesgo de embolia fueron aleatorizados a warfarina (INR 2 - 3) vs. dabigatrán etexilato $150 \mathrm{mg}$ dos veces al día, por un periodo de dos años; se consideraron en alto riesgo embólico los pacientes mayores de 75 años o entre 65 y 74 años con diabetes, enfermedad arterial coronaria, hipertensión arterial, historia de ataque cerebro-vascular previo o isquemia cerebral transitoria, fracción de expulsión del ventrículo izquierdo menor de $40 \%$ o clase funcional NYHA II. Se excluyeron pacientes con depuración de creatinina menor de $30 \mathrm{ml}$ por minuto o enfermedad hepática activa. El punto final primario fue la incidencia de ataque cerebrovascular o embolia sistémica. Se demostró la no inferioridad de las dosis de 110 y $150 \mathrm{mg}$ de dabigatrán etexilato respecto a la warfarina, con la dosis de $150 \mathrm{mg}$ dos veces al día, siendo esta significativamente superior para prevenir ataque cerebro-vascular y embolia sistémica que la warfarina. La dosis de $110 \mathrm{mg}$ dos veces al día no demostró diferencia respecto a la warfarina en este punto. El beneficio clínico (compuesto de la incidencia de ataque cerebrovascular, embolia sistémica o pulmonar, infarto al miocardio y muerte) fue superior con dabigatrán a la dosis de $150 \mathrm{mg}$ dos veces al día. En este estudio no hubo diferencias en cuanto a incidencia de sangrado mayor (reducción $>50 \mathrm{~g} / \mathrm{L}$ de hemoglobina o necesidad de transfusión $>4$ unidades de sangre) con dosis de $150 \mathrm{mg}$ dos veces día vs. Warfarina para un INR de 2 - 3, pero sí más episodios de sangrado gastrointestinal ${ }^{17}$. La dosis de $110 \mathrm{mg}$ dos veces día tiene menor incidencia de sangrado que la de $150 \mathrm{mg}$ dos veces día o warfarina.

\section{Tolerabilidad}

A la dosis usual de 110 o $150 \mathrm{mg}$ dos veces al día, la única diferencia según los estudios RE-LY y PETRO fue la dispepsia (mayor con dabigatrán etexilato que con warfarina). Las reacciones de hipersensibilidad fueron infrecuentes $(0,1 \%)$, en tanto que se registró un 35\% de reacciones adversas gastrointestinales. No hubo diferencia en cuanto a hepatotoxicidad comparado con warfarina. 


\section{Consideraciones farmacoeconómicas}

En el estudio de costo-efectividad de dabigatrán vs. warfarina realizado en Colombia, los pacientes tratados con dabigatrán 150 y $110 \mathrm{mg}$ ganaron en promedio 0,37 y 0,23 años de vida respectivamente, o 0,55 y 0,43 años de vida ajustados por calidad (QALY, su sigla en inglés) ${ }^{18}$. La razón de costo-efectividad incremental para dabigatrán $150 \mathrm{mg}$ fue \$23.078.506 por QALY ganado, mientras que para la dosis de $110 \mathrm{mg}$ fue de $\$ 34.186 .731$, cifras que indican que los resultados de la evaluación de costo-efectividad de dabigatrán en el tratamiento de la fibrilación auricular no valvular en Colombia muestran un incremento de los QALY ganados con dabigatrán para los dos regímenes de dosificación disponibles (150 y $110 \mathrm{mg}$ ), en comparación con warfarina. Esta ganancia en QALY es atribuible a la disminución de la incidencia de desenlaces clínicos adversos, tanto mortales como discapacitantes, y por ende a un aumento de la sobrevida y calidad de vida de los pacientes con fibrilación auricular. Por tanto, dabigatrán (ambas dosis), comparado con warfarina, es una alternativa costo-efectiva para el tratamiento de la fibrilación auricular no valvular en Colombia.

\section{Dosis y administración}

Se recomienda una dosis oral de $150 \mathrm{mg}$ dos veces al día en pacientes con depuración de creatinina $>30 \mathrm{~mL} / \mathrm{min}$ y de $75 \mathrm{mg}$ dos veces al día (solo en los Estados Unidos) en pacientes con depuración de creatinina $(\mathrm{CrCl}$, su sigla en inglés) entre 15 - $30 \mathrm{ml} / \mathrm{min}$; no se sugiere su uso cuando es menor de $15 \mathrm{ml} / \mathrm{min}$. En pacientes mayores de 80 años, se debe disminuir la dosis a $110 \mathrm{mg}$ dos veces al día. No hay diferencias claras en cuanto al nivel de recomendación acerca del uso de dabigatrán si se trata de prevención primaria o secundaria o de acuerdo con el tiempo de duración de la fibrilación auricular (paroxística, persistente, permanente), así que las dosis utilizadas deben ser las mismas.

\section{Recomendaciones}

1. Dabigatrán (o cualquier otro NACO) está indicado para la prevención primaria o secundaria de ataque cerebrovascular en pacientes con fibrilación auricular no valvular con alto riesgo de cardioembolia $\left(\mathrm{CHADS}_{2} \geq 10\right.$ $\mathrm{CHA}_{2} \mathrm{DS}_{2} \mathrm{VASC} \geq 1$ ) (recomendación clase I, nivel de evidencia $A$ ).

2. En pacientes menores de 80 años, la dosis de dabigatrán es de $150 \mathrm{mg}$ VO cada 12 horas; en pacientes $\geq 80$ años se recomienda utilizar una dosis de $110 \mathrm{mg}$ VO cada 12 horas (recomendación clase I, nivel de evidencia A).

3. En pacientes con alto riesgo de sangrado (definido como un puntaje de HAS-BLED $\geq 3$ ) se puede considerar reducir la dosis de dabigatrán a $110 \mathrm{mg}$ Vo cada 12 horas (recomendación clase lla, nivel de evidencia $\mathrm{C}$ ).

4. En pacientes con fibrilación auricular no valvular y enfermedad renal crónica estadio $v$ o en terapia dialítica, no debe administrarse dabigatrán. En estos casos se prefiere el uso de warfarina (recomendación clase III, nivel de evidencia C).

\section{Rivaroxabán}

Al igual que los demás inhibidores del factor Xa (apixabán, edoxabán, betrixabán), evita el paso de protrombina a trombina. Es un inhibidor selectivo del factor Xa que no requiere la antitrombina como cofactor y ejerce efecto en el factor Xa libre, en el que se encuentra adherido al trombo y en el del complejo protrombinasa ${ }^{19,20}$.

\section{Propiedades farmacocinéticas}

El efecto pico es de 2 a 4 horas posingesta, con una vida media de eliminación de 5-9 horas; aumenta hasta 13 horas en ancianos, no es afectado por los antiácidos y su absorción se eleva al consumirse con los alimentos. Su biodisponibilidad es del $66 \%$ y aumenta con los alimentos al $80 \%$. Dos tercios del medicamento tienen metabolismo hepático mediante oxidación vía citocromos e hidrólisis y hasta el 70\% es de eliminación renal. El 30-33\% de la dosis administrada es excretada por el riñón como sustancia activa sin variación en la orina, principalmente por secreción renal activa. Las otras dos terceras partes experimentan degradación metabólica, y la mitad (33\% de la dosis total) es eliminada de forma inactiva por el riñón, mientras que la otra mitad es eliminada por la vía hepatobiliar.

\section{Interacciones farmacológicas}

La interacción con los inhibidores de la CYP3A4 (ketoconazol, claritromicina): puede aumentar el área bajo la curva $^{21}$ y con los inductores de la CYP3A4 (rifampicina) disminuye el AUC. El rivaroxabán es sustrato de la glicoproteína $\mathrm{P}$, por lo tanto se debe vigilar la interacción con medicamentos inhibidores como: amiodarona, dronedarona, verapamilo y con inductores como la rifampicina. Debido a la necesidad potencial de ajuste de dosis, debe usarse con precaución en pacientes con depuración de creatinina entre 15 y $50 \mathrm{ml}$.

\section{Eficacia terapéutica para la prevención del ataque cerebro-vascular y la embolia sistémica en fibrilación auricular}

El estudio ROCKET AF evaluó la eficacia y seguridad de rivaroxabán comparado con warfarina para la prevención de embolia en pacientes con fibrilación auricular no valvular $^{22}$. Cerca del $90 \%$ de los pacientes evaluados tenían un puntaje de $\mathrm{CHADS}_{2} \geq 3$. El estudio demostró la no inferioridad respecto a warfarina para la prevención del ataque cerebro-vascular y embolia sistémica, sin lograr demostrar superioridad en el análisis por intención de tratar. En el desenlace de seguridad hubo una tasa menor de sangrado intracerebral y hemorragias fatales, las cuales fueron estadísticamente significativas $(p=0,02$ y 0,003, respectivamente). Sin embargo, con base en los análisis preclínicos la FDA aprobó el uso de rivaroxabán con precaución en pacientes con depuración de creatinina entre 15 y 30.

Estudios que evalúan pacientes de la práctica clínica cotidiana han reproducido los resultados de los estudios clínicos. 
El RELIEF, un estudio retrospectivo llevado a cabo en Alemania, evaluó un desenlace combinado de eventos isquémicos y hemorrágicos, y halló menor incidencia en los pacientes anticoagulados con rivaroxabán que en aquellos anticoagulados con warfarina ${ }^{23}$.

En estudios prospectivos observacionales en pacientes de la práctica clínica cotidiana como el reciente estudio XANTUS, los datos de seguridad y eficacia fueron menores que en el ROCKET, con tasas de sangrado y embolia de 2,1 vs. 3,6 y de 0,8 vs, 2,1 respectivamente. Es importante resaltar que en este estudio, los médicos tratantes decidieron anticoagular con rivaroxaban a pacientes con menos riesgo según la escala de CHADS comparado con ROCKET (2 vs. 3,6 respectivamente) ${ }^{24}$.

Según datos del registro Dresden, la adherencia y persistencia de los pacientes anticoagulados con rivaroxabán fueron estadísticamente mejores que en aquellos anticoagulados con dabigatrán y warfarina ${ }^{30}$.

\section{Indicaciones}

\section{Prevención primaria de ataque cerebro-vascular cardioembólico en pacientes con fibrilación auricular no valvular}

Las recomendaciones para anticoagulación difieren en su nivel de recomendación de acuerdo con las guías analizadas. Para americanos ${ }^{25}$ y canadienses $^{26}$, se sugiere anticoagulación en pacientes con $\mathrm{CHADS}_{2} \geq 1$ o $\mathrm{CHA}_{2} \mathrm{DS}_{2}$-VASc $\geq$ 1 con recomendaciones clase I. Para los europeos ${ }^{27}$, la anticoagulación en pacientes con $\mathrm{CHA}_{2} \mathrm{DS}_{2}-\mathrm{VASC} \geq 2$ tiene recomendación tipo I, mientras que la anticoagulación en pacientes con $\mathrm{CHA}_{2} \mathrm{DS}_{2}-\mathrm{VASC}=1$ tiene recomendación Ila. $\mathrm{La}$ dosis de rivaroxabán para la prevención de embolia en fibrilación auricular (de acuerdo con los resultados del ROCKET) es de $20 \mathrm{mg}$ al día, ajustada a $15 \mathrm{mg}$ en pacientes con $\mathrm{CrCl}$ $30-49 \mathrm{~mL} / \mathrm{min}$. En el ROCKET AF no se evaluaron pacientes con enfermedad renal crónica estadio 4 o 5. Sin embargo, con base en los análisis preclínicos la FDA aprobó el uso de rivaroxabán con precaución en pacientes con depuración de creatinina entre 15 y 30 , en tanto que no está indicado en aquellos con depuración de creatinina menor de 15. La edad como criterio aislado no debe utilizarse para modificar la dosis del medicamento. Un subanálisis del ROCKET $\mathrm{AF}^{28}$ encontró que la eficacia y seguridad del rivaroxabán es comparable con warfarina entre los pacientes mayores y menores de 75 años, con mayores tasas de sangrado gastrointestinal.

El rivaroxabán a dosis de $20 \mathrm{mg}$ día está indicado para la prevención primaria del ataque cerebro-vascular en pacientes con alto riesgo de cardioembolia $\left(\mathrm{CHADS}_{2} \geq 10\right.$ $\mathrm{CHA}_{2} \mathrm{DS}_{2}-\mathrm{VASC} \geq 1$ ). Las guías americanas sugieren warfarina o NACO como opciones equivalentes ${ }^{25}$, y las guías europeas los sugieren como opciones equivalentes en pacientes con $\mathrm{CHA}_{2} \mathrm{DS}_{2}$-VASc $\geq 2^{27}$. Las guías canadienses proponen los nuevos anticoagulantes orales (NACO) sobre la warfarina en pacientes con $\mathrm{CHADS}_{2} \geq 1^{26}$. Dado que no existen comparaciones directas entre los NACO, la selección del medicamento debe individualizarse teniendo en cuenta factores de riesgo, comorbilidades, costos, tolerabilidad, interacciones, función renal, entre otros.

\section{Recomendación}

1. Se sugiere el uso de rivaroxabán (o cualquier otro NACO) en el manejo de pacientes con fibrilación auricular no valvular para la prevención primaria o secundaria de embolia cerebral (recomendación clase ।, nivel de evidencia A).

2. La dosis de rivaroxabán es de $20 \mathrm{mg}$ Vo cada 24 horas. Se sugiere el uso de una dosis ajustada de rivaroxabán de $15 \mathrm{mg}$ cada 24 horas en pacientes con compromiso moderado de la función renal, definida como $\mathrm{CrCl}$ $30-49 \mathrm{~mL} / \mathrm{min}$ (recomendación clase lla, nivel de evidencia C).

3. En pacientes con fibrilación auricular no valvular y enfermedad renal crónica estadio $v$ o en terapia dialítica, no debe administrarse rivaroxabán. En estos casos se sugiere el uso de warfarina ajustada para un INR de 2-3 (recomendación clase III, nivel de evidencia C).

\section{Apixabán}

Es un inhibidor selectivo del factor $\mathrm{Xa}$, que tiene efecto tanto en el factor Xa libre en plasma como en el que se encuentra unido al complejo protrombinasa presente en la superficie del trombo en formación. Mediante la inhibición del factor $\mathrm{Xa}$, disminuye en modo logarítmico la formación de trombina y por consiguiente la del trombo (la inhibición de una molécula de factor $\mathrm{Xa}$ tiene como consecuencia la inhibición en la generación de 1.000 moléculas de trombina). Es responsable de la inhibición plaquetaria en forma indirecta al reducir los niveles de trombina y la activación plaquetaria mediada por ésta. El apixabán se une de forma directa al factor Xa presente en el coágulo en formación, generando una inhibición reversible de la activación plaquetaria y del coágulo de fibrina en formación ${ }^{29-33}$. La biodisponibilidad del apixabán es cercana al $50 \%$ en individuos sanos, y no se ve afectada por la alimentación, el género o la raza ${ }^{32,34}$. Alcanza una concentración máxima en sangre entre 3 y 4 horas después de su administración por vía oral, una vida media de 8 a 15 horas y una tasa de unión a proteínas del $87 \%$. Sufre escasa transformación metabólica y su eliminación sin cambios es cercana al 70 , por vías de predominio hepático; solo un $27 \%$ se excreta por vía renal ${ }^{31}$. Cerca de un $30 \%$ del medicamento sufre algún grado de trasformación mediante el sistema de los citocromos (CYP3A4 / 5 y en menor medida por CYP1A2 y CYP2J2), sin que esto signifique que esta molécula tenga un rol en la inducción o inhibición del citocromo P450. En términos generales, la función renal altera poco los parámetros farmacocinéticos de la molécula. Solo en individuos con depuración de creatinina menor de $30 \mathrm{ml} / \mathrm{min}$ se puede aumentar hasta en un $44 \%$ el área bajo la curva de concentración del medicamento, de ahí que sea posible que se presente un aumento en la exposición al fármaco en este grupo.

Con base en el concepto previo y la no inclusión en los estudios clínicos fase III de pacientes con depuración menor de $15 \mathrm{ml} / \mathrm{min}$ y otros de $30 \mathrm{ml} / \mathrm{min}$ o que estén en programas de terapia de reemplazo renal, esta molécula no está indicada para su uso en pacientes con insuficiencia renal estadio Iv o v o depuración menor a $15 \mathrm{ml} / \mathrm{min}$. 
Las condiciones extremas de peso (>120 kg o menor de $50 \mathrm{~kg}$ ) reducen o incrementan respectivamente la concentración del medicamento y deben tenerse en cuenta al formular la dosis; adicionalmente, se demostró que los volúmenes de distribución se afectan con la edad y generan así mayor concentración del medicamento en pacientes de más de 80 años. De lo anterior se deriva la reducción en la dosis en un $50 \%$ en los estudios AVERROES y ARISTOTLE ${ }^{7,8}$, en pacientes que cumplieran al menos dos de las siguientes condiciones: peso menor a $60 \mathrm{~kg}$, edad mayor a 80 años 0 creatinina mayor a $1,5 \mathrm{mg} / \mathrm{dl}$.

Los inhibidores del factor Xa pueden modificar en forma sutil los valores de tiempo de protrombina (PT) y tiempo parcial de tromboplastina (PTT) en diferente proporción. El PTT no puede usarse para evaluación del efecto antitrombótico debido a su escasa modificación y amplia variabilidad de acuerdo con el tipo de análisis realizado.

\section{Eficacia terapéutica para la prevención de ataque cerebro-vascular y embolia sistémica en fibrilación auricular}

En el estudio ARISTOTLE se aleatorizaron más de 18.000 pacientes con índice $\mathrm{CHADS}_{2}$ promedio de 2,1 puntos a una dosis de $5 \mathrm{mg}$ cada 12 horas frente a warfarina a una dosis de $5 \mathrm{mg}$ / día (ajustada para mantener un INR entre 2 y 3); la dosis de apixabán se ajustó a 2,5 mg cada 12 horas en pacientes con al menos dos de las siguientes condiciones: peso menor de $60 \mathrm{~kg}$, creatinina mayor $1,5 \mathrm{mg} / \mathrm{dl}$ y edad mayor de 80 años $^{32-35}$. Se excluyeron pacientes con fibrilación auricular de causa secundaria, estenosis mitral moderada a severa, condiciones diferentes a fibrilación auricular que hicieran necesaria la anticoagulación (prótesis valvulares cardiacas), ataque cerebro-vascular en los siete días previos, necesidad de una dosis de ASA mayor a $165 \mathrm{mg} /$ día o uso de terapia antiplaquetaria dual (ASA/clopidogrel) y pacientes con disfunción renal severa, definida como depuración calculada de creatinina menor de $25 \mathrm{ml} / \mathrm{min}$, o creatinina mayor a 2,5 mg/dl.

Con un seguimiento a 1,8 años se demostró una reducción en el punto final primario (tasa de eventos de ataque cerebro-vascular isquémico, hemorrágico y embolia sistémica), en cuyo caso la intervención con apixabán no inferior e incluso superior a la terapia estándar. Adicionalmente, este demostró reducción en el riesgo de sangrado y mortalidad por todas las causas. En este estudio el beneficio del apixabán fue independiente del tipo de fibrilación auricular (paroxística, persistente o permanente), el índice de riesgo $\left(\mathrm{CHADS}_{2}-\mathrm{CHADS}_{2}-\mathrm{VAS}_{\mathrm{C}}\right)$ e incluso la historia previa de ataque cerebro-vascular.

En el estudio AVERROES se comparó el apixabán frente a ASA en pacientes con indicación de anticoagulación considerados no candidatos a warfarina ${ }^{36}$. Este culminó temprano luego del primer análisis de seguridad, momento en el cual el apixabán ya había demostrado una reducción marcada del riesgo de ataque cerebro-vascular y de embolia sistémica. La molécula tiene un perfil de seguridad (definida como la tasa de sangrados asociada al uso del fármaco) muy similar al del ASA, concluyéndose así que su uso es seguro y efectivo aun en poblaciones que previamente no podían ser anticoaguladas y a quienes se les ofrecía una terapia insuficiente.

\section{Edoxabán}

Es un inhibidor selectivo del factor $\mathrm{Xa}$, de rápida absorción después de una dosis oral y biodisponibilidad del 62\% que no se ve afectada con la ingesta de alimentos ${ }^{37,38}$. El $72 \%$ del fármaco se elimina sin modificaciones, principalmente en heces (62\%) y vía renal (35\%), y su metabolismo hepático es mínimo $(4 \%)^{39,40}$. Su eliminación fecal es dependiente del mecanismo de glicoproteína $\mathrm{P}$, por lo cual sus inhibidores tendrán un impacto significativo en la exposición al fármaco. Por esta razón, está contraindicado su uso concomitante con medicamentos como antibióticos macrólidos (eritromicina, azitromicina, claritromicina), antimicóticos azoles (ketoconazol e itraconazol), ritonavir y ciclosporina ${ }^{38,41}$. Por su parte, el verapamilo, la quinidina y la dronedarona (mas no la amiodarona) se asocian con aumento de las concentraciones séricas (también mediante la inhibición de la glicoproteína $\mathrm{P}$ ) que obliga a modificar la dosis, disminuyéndola en un $50 \%{ }^{42,43}$.

\section{Eficacia terapéutica para la prevención de ataque cerebro-vascular y embolia sistémica en fibrilación auricular}

El ENGAGE AF-TIMI 48 fue un estudio aleatorizado, doble ciego con doble simulación y de no inferioridad que evaluó la utilidad y seguridad del edoxabán en la prevención de embolia en pacientes con fibrilación auricular ${ }^{41}$. Se aleatorizaron 21.105 pacientes con fibrilación auricular y $\mathrm{CHADS}_{2}$ $\geq 2$ a warfarina (ajustada para lograr un INR de 2-3) o uno de dos esquemas de dosificación de edoxabán. Se estudiaron dosis de $60 \mathrm{mg}$ y de $30 \mathrm{mg}$ VO cada día de edoxabán, los cuales fueron reducidos a la mitad (a 30 o $15 \mathrm{mg}$ VO cada día) en aquellos pacientes con uno de los siguientes: peso $\leq$ $60 \mathrm{~kg}$, depuración de creatinina $30-50 \mathrm{ml} / \mathrm{min}$ (se excluyeron pacientes con una depuración de creatinina $\leq 30 \mathrm{ml} / \mathrm{min}$ ) 0 uso de un inhibidor potente de la glicoproteína $\mathrm{P}$ (verapamilo, dronedarona, quinidina).

Se logró demostrar la no inferioridad en prevención de embolia para la dosis de $30 \mathrm{mg}$ de edoxabán y superioridad para la dosis de $60 \mathrm{mg}$ respecto a warfarina, con menor tasa de hemorragia intracerebral y sangrado mayor para ambas dosis de edoxabán. El riesgo de sangrado gastrointestinal fue mayor en los pacientes que recibieron $60 \mathrm{mg}$ al día de edoxabán respecto a warfarina $(1,51 \%$ vs. $1,23 \%$; HR $1,23[1,02 / 1,05])$; el riesgo fue menor en pacientes que recibieron edoxabán $30 \mathrm{mg}(0,82 \%$; HR 0,67 $[0,53-0,83])$.

\section{Dosificación}

En la actualidad, la dosis de edoxabán recomendada para la prevención de embolia en pacientes con fibrilación auricular no valvular es de $60 \mathrm{mg}$ VO cada día. Dado el aumento de exposición en pacientes con un peso inferior a $60 \mathrm{~kg}$, depuración de creatinina $30-50 \mathrm{~mL} / \mathrm{min}$ y exposición a fármacos que inhiban la glicoproteína $\mathrm{P}$ (verapamilo, quinidina, dronedarona) se recomienda disminuir su dosis a la mitad en estos $\operatorname{casos}^{41,44}$. Vale la pena resaltar que la evaluación de subgrupos ha reportado aumento en la tasa de fenómenos 
embólicos en pacientes con $\mathrm{CrCl} \geq 95 \mathrm{~mL} / \mathrm{min}$, posiblemente asociado con disminución en la exposición al fármaco debido a mayor eliminación renal ${ }^{45}$.

\section{Recomendaciones}

1. Se prefiere el uso de edoxabán (o cualquier otro NACO) como primera opción en pacientes con fibrilación auricular no valvular que no tengan contraindicación para el uso de este medicamento y que no venían recibiendo previamente terapia anticoagulante con warfarina (recomendación clase I, nivel de evidencia A).

2. En pacientes que vienen recibiendo warfarina con un tiempo en rango terapéutico menor del $60 \%$, se sugiere el uso de edoxabán (o cualquier otro NACO) como estrategia efectiva para la prevención de eventos embólicos (recomendación clase I, nivel de evidencia A).

3. En pacientes que vienen recibiendo warfarina con un adecuado control y un tiempo en rango terapéutico mayor al $60 \%$, es posible considerar el cambio de esta a edoxabán si se considera que el riesgo de sangrado es significativo (recomendación clase lla, nivel de evidencia A).

4. Se sugiere el uso de edoxabán a una dosis de $60 \mathrm{mg}$ VO cada día en pacientes con fibrilación auricular no valvular que no tienen condiciones que se asocien con un aumento en la exposición al fármaco (recomendación clase I, nivel de evidencia A).

5. En pacientes con una o más condiciones asociadas a un aumento en la exposición a edoxabán (peso menor a $60 \mathrm{~kg}$, depuración de creatinina de $30-50 \mathrm{~mL} / \mathrm{min}$ o que estén tomando verapamilo, quinidina o dronedarona) se sugiere una dosis de edoxabán de $30 \mathrm{mg}$ Vo cada día (recomendación clase I, nivel de evidencia A).

6. En pacientes con depuración de creatinina 15$30 \mathrm{~mL} / \mathrm{min}$, se puede considerar el uso de edoxabán a una dosis de $15 \mathrm{mg}$ VO cada día cuando no existen otras alternativas de manejo (recomendación clase llb, nivel de evidencia $B$ ).

7. En pacientes con depuración de creatinina $\leq 15 \mathrm{~mL} / \mathrm{min}$ o en terapia de reemplazo renal no se recomienda el uso de edoxabán (recomendación clase III, nivel de evidencia A).

8. Se sugiere evitar el uso de edoxabán en pacientes con depuración de creatinina $\geq 95 \mathrm{~mL} / \mathrm{min}$ (recomendación clase lla, nivel de evidencia B).

9. En pacientes en tratamiento con edoxabán y que serán cambiados a otro NACO, se sugiere iniciar el nuevo medicamento al día siguiente de haber suspendido el edoxabán (recomendación clase I, nivel de evidencia B).

10. En pacientes en tratamiento con edoxabán y que serán cambiados a warfarina, se sugiere continuar con una fase de transición utilizando dosis reducidas de edoxabán $(30 \mathrm{mg}$ VO cada día si el paciente no ha requerido disminución de la dosis o de $15 \mathrm{mg}$ si ha requerido disminución de la dosis) hasta por 14 días o hasta que se logre un INR $\geq 2$. Durante esta fase de transición se sugiere monitorización estricta diaria del INR con el fin de identificar el momento exacto en el cual se alcanza el rango terapéutico (recomendación clase I, nivel de evidencia A).

\section{Conclusión}

Muchos pacientes con fibrilación auricular son candidatos a terapia anticoagulante con el objetivo de disminuir el riesgo de ataque cerebro-vascular y tromboembolia sistémica. Independiente del riesgo de sangrado, esta terapia tiene mayor beneficio que riesgos. El uso de los anticoagulantes directos proporciona gran beneficio en cuanto a la prevención de eventos tromboembólicos y genera menores tasas de eventos hemorrágicos. Aunque las diferencias estadísticas entre los anticoagulantes son estrechas y en algunos casos no significativas, se podría recomendar que la elección de uno u otro en determinados casos se haga con base en la edad y la presencia o ausencia de ciertas comorbilidades como falla renal severa, enfermedad coronaria, embolia previa, riesgo de sangrado, medicamentos concomitantes, función hepática y compromiso valvular mitral.

\section{Conflicto de intereses}

Los autores declaran no tener conflicto de intereses.

\section{Bibliografía}

1. Amin AN, Jhaveri M, Lin J. Incremental cost burden to US healthcare payers of atrial fibrillation/atrial flutter patients with additional risk factors. Advances in Therapy. 2011;28:907-26.

2. Wolf PA, Dawber TR, Thomas HE, et al. Epidemiologic assessment of chronic atrial fibrillation and risk of stroke: the Framingham study. Neurology. 1978;28:973-7.

3. Gage BF, Waterman AD, Shannon W, et al. Validation of clinical classification schemes for predicting stroke: results from the National Registry of Atrial Fibrillation. JAMA. 2001;285:2864-70.

4. Lip GY, Nieuwlaat R, Pisters R, et al. Refining clinical risk stratification for predicting stroke and thromboembolism in atrial fibrillation using a novel risk factor-based approach: the euro heart survey on atrial fibrillation. Chest. 2010;137:263-72.

5. Stroke Prevention in Atrial Fibrillation Study. Final results. Circulation. 1991;84:527-39.

6. Ezekowitz MD, Bridgers SL, James KE, et al. Warfarin in the prevention of stroke associated with nonrheumatic atrial fibrillation. Veterans Affairs Stroke Prevention in Nonrheumatic Atrial Fibrillation Investigators. New Eng J Med. 1992;327:1406-12.

7. Warfarin versus aspirin for prevention of thromboembolism in atrial fibrillation: Stroke Prevention in Atrial Fibrillation II Study. Lancet (London, England). 1994;343:687-91.

8. Albers GW, Atrial fibrillation and stroke. Three new studies, three remaining questions. Arch Int Med. 1994;154:1443-8.

9. Laupacis A, Albers G, Dunn M, Feinberg W. Antithrombotic therapy in atrial fibrillation. Chest. 1992;102 4 Suppl:426s-33s.

10. Tajer C, Ceresetto J, Bottaro FJ, et al. Assessment of the Quality of Chronic Anticoagulation Control With Time in Therapeutic Range in Atrial Fibrillation Patients Treated With Vitamin K Antagonists by Hemostasis Specialists: The TERRA Registry: Tiempo en rango en la República Argentina. Clin Appl Thromb Hemost. 2016, http://dx.doi.org/10.1177/1076029615623378.

11. Ammollo CT, Semeraro F, Incampo F, et al. Dabigatran enhances clot susceptibility to fibrinolysis by mechanisms dependent on and independent of thrombin-activatable fibrinolysis inhibitor. J Thromb Haemost. 2010;8:790-8.

12. Sanford M, Plosker GL. Dabigatran etexilate. Drugs. 2008;68: 1699-709. 
13. Stangier J, Rathgen K, Stahle H, Mazur D. Influence of renal impairment on the pharmacokinetics and pharmacodynamics of oral dabigatran etexilate: an open-label, parallel-group, singlecentre study. Clinical pharmacokinetics. 2010;49:259-68.

14. Garnock-Jones KP. Dabigatran etexilate: a review of its use in the prevention of stroke and systemic embolism in patients with atrial fibrillation. Am J Cardiovac Drugs: drugs, devices, and other interventions. 2011;11:57-72.

15. Ezekowitz MD, Reilly PA, Nehmiz G, et al. Dabigatran with or without concomitant aspirin compared with warfarin alone in patients with nonvalvular atrial fibrillation (PETRO Study). Am J Cardiol. 2007;100:1419-26.

16. Connolly SJ, Ezekowitz MD, Yusuf S, et al. Dabigatran versus warfarin in patients with atrial fibrillation. New Eng J Med. 2009;361:1139-51

17. Connolly SJ, Ezekowitz MD, Yusuf S, et al. Newly identified events in the RE-LY trial. New Eng J Med. 2010;363:1875-6.

18. Triana JJ, Castañeda C, Parada L, et al. Costo-efectividad de dabigatrán comparado con warfarina para el tratamiento de pacientes con fibrilación auricular no valvular. Rev Colomb Cardiol. 2016;23:82-6.

19. Schirmer SH, Baumhakel M, Neuberger HR, et al. Novel anticoagulants for stroke prevention in atrial fibrillation: current clinical evidence and future developments. J Am Coll Cardiol. 2010;56:2067-76.

20. Aristizábal J, Restrepo A, Uribe W, et al. Consideraciones prácticas para el uso de los nuevos anticoagulantes orales. Rev Colomb Cardiol. 2012;19:7.

21. Fauchier L, Clementy N, Saint-Etienne C, et al. Efficacy of new oral anticoagulants in patients with atrial fibrillation previously treated with warfarin: a meta-analysis of randomized controlled trials. Int J Cardiol. 2014;173:122-4.

22. Patel MR, Mahaffey KW, Garg J, et al. Rivaroxaban versus warfarin in nonvalvular atrial fibrillation. New Eng J Med. 2011;365:883-91.

23. Coleman $\mathrm{Cl}$, Antz M, Ehlken B, Evers T. REal-Llfe Evidence of stroke prevention in patients with atrial Fibrillation - The RELIEF study. Int J Cardiol. 2016;203:882-4.

24. Camm AJ, Amarenco P, Haas S, et al. XANTUS: a real-world, prospective, observational study of patients treated with rivaroxaban for stroke prevention in atrial fibrillation. Eur Heart J. 2016;37:1145-53.

25. January CT, Wann LS, Alpert JS, et al. 2014 AHA/ACC/HRS guideline for the management of patients with atrial fibrillation: a report of the American College of Cardiology/American Heart Association Task Force on Practice Guidelines and the Heart Rhythm Society. J Am Coll Cardiol. 2014;64:e1-76.

26. Verma A, Cairns JA, Mitchell LB, et al. 2014 focused update of the Canadian Cardiovascular Society Guidelines for the management of atrial fibrillation. Can J Cardiol. 2014;30:1114-30.

27. Camm AJ, Lip GY, De Caterina R, et al. 2012 focused update of the ESC Guidelines for the management of atrial fibrillation: an update of the 2010 ESC Guidelines for the management of atrial fibrillation-developed with the special contribution of the European Heart Rhythm Association. Europace. 2012;14:1385-413.

28. Halperin JL, Hankey GJ, Wojdyla DM, et al. Efficacy and safety of rivaroxaban compared with warfarin among elderly patients with nonvalvular atrial fibrillation in the Rivaroxaban Once Daily, Oral, Direct Factor Xa Inhibition Compared With Vitamin K Antagonism for Prevention of Stroke and Embolism Trial in Atrial Fibrillation (ROCKET AF). Circulation. 2014;130:138-46.
29. Raghavan N, Frost CE, Yu Z, et al. Apixaban metabolism and pharmacokinetics after oral administration to humans. Drug Metab Dispos. 2009;37:74-81.

30. Turpie AG. Oral, direct factor Xa inhibitors in development for the prevention and treatment of thromboembolic diseases. Arteriosclerosis, thrombosis, and vascular biology. 2007;27:1238-47.

31. Wong PC, Pinto DJ, Zhang D. Preclinical discovery of apixaban, a direct and orally bioavailable factor Xa inhibitor. J Thrombosis and Thrombolysis. 2011;31:478-92.

32. Frost C, Wang J, Nepal S, et al. Apixaban, an oral, direct factor Xa inhibitor: single dose safety, pharmacokinetics, pharmacodynamics and food effect in healthy subjects. Brit J Clin Pharmacol. 2013;75:476-87.

33. Frost C, Yu Z, Moore K, et al. Apixaban, an oral direct factor Xa inhibitor: multiple-dose safety, pharmacokinetics, and pharmacodynamics in healthy subjects. J Thromb Haemost. 2007;5 Suppl 2:PM-M664.

34. Frost C, Yu Z, Shenker A, Nepal S, editors. Food does not affect the pharmacokinetics of apixabán, an oral factor Xa inhibitor. $9^{\text {th }}$. World Conf Clin Pharmacol Ther July; 2008.

35. Granger CB, Alexander JH, McMurray JJ, et al. Apixaban versus warfarin in patients with atrial fibrillation. New Eng J Med. 2011;365:981-92.

36. Connolly SJ, Eikelboom J, Joyner C, et al. Apixaban in patients with atrial fibrillation. New Eng J Med. 2011;364:806-17.

37. Mendell J, Tachibana M, Shi M, Kunitada S. Effects of food on the pharmacokinetics of edoxaban, an oral direct factor Xa inhibitor, in healthy volunteers. J Clin Pharmacol. 2011;51:687-94.

38. Bounameaux H, Camm AJ. Edoxaban: an update on the new oral direct factor Xa inhibitor. Drugs. 2014;74:1209-31.

39. Ogata K, Mendell-Harary J, Tachibana M, et al. Clinical safety, tolerability, pharmacokinetics, and pharmacodynamics of the novel factor Xa inhibitor edoxaban in healthy volunteers. J Clin Pharmacol. 2010;50:743-53.

40. Bathala MS, Masumoto H, Oguma T, et al. Pharmacokinetics, biotransformation, and mass balance of edoxaban, a selective, direct factor Xa inhibitor, in humans. Drug metabolism and disposition: the biological fate of chemicals. 2012;40:2250-5.

41. Giugliano RP, Ruff CT, Braunwald E, et al. Edoxaban versus warfarin in patients with atrial fibrillation. New Eng J Med. 2013;369:2093-104.

42. Mendell J, Zahir H, Matsushima N, et al. Drug-drug interaction studies of cardiovascular drugs involving P-glycoprotein, an efflux transporter, on the pharmacokinetics of edoxaban, an oral factor Xa inhibitor. Am J Cardiovasc Drugs: drugs, devices, and other interventions. 2013;13:331-42.

43. Matsushima N, Lee F, Sato T, et al. Bioavailability and safety of the factor Xa inhibitor edoxaban and the effects of quinidine in healthy subjects. Clin Pharmacol Drug Dev. 2013;2:358-66.

44. Yamashita T, Koretsune Y, Yasaka M, et al. Randomized, multicenter, warfarin-controlled phase II study of edoxaban in Japanese patients with non-valvular atrial fibrillation. Circulation journal: official journal of the Japanese Circulation Society. 2012;76:1840-7.

45. SAVAYSA (edoxaban) Tablets. FDA Draft Briefing Document for the Cardiovascular and Renal Drugs Advisory Committee. US Food and Drug Administration [Acceso 30 Oct 2014]. Disponible en: http://www.fda.gov/downloads/AdvisoryCommittees/ CommitteesMeetingMaterials/Drugs/CardiovascularandRenal DrugsAdvisoryCommittee/UCM420704.pdf2014 